\title{
Looking for volatile and mineralogy in carbonaceous chondrites.
}

\author{
ARAVENA-GONZÁLEZ, S. ${ }^{1}$, MONCADA, D. ${ }^{1}$, \\ VARELA, M. E. ${ }^{2}$, MORLOK, A. ${ }^{3} \&$ MARTÍNEZ, R. ${ }^{4}$ \\ ${ }^{1}$ Department of Geology, University of Chile, Plaza Ercilla \\ 803, Santiago, Chile, samanta.aravena@gmail.com, \\ dmoncada@ing.uchile.cl \\ ${ }^{2}$ Av. España Sur 1512, San Juan, Argentina, \\ eugeniavarela@conicet.gov.ar \\ ${ }^{3}$ Wilhelm-Klemm-Str.10 48149 Münster, Germay, \\ morlok70@me.com \\ ${ }^{4}$ Tocopilla 101, San Pedro de Atacama, Antofagasta, Chile, \\ rodrigo@museodelmeteorito.cl
}

The carbonaceous chondrites might be containing the oldest organic molecules that fall to the Earth. These specimens are the natural laboratory that contains organic molecules product of the chemical evolution of our solar system. About $4 \%$ of the $\sim 70,000$ meteorites recorded according to the Meteoritical Bulletin (accessed in February 2020) correspond to carbonaceous chondrites in the world collection. Based on studies of melt inclusions assemblages (MIA) in olivines, their formation in chondrules and meteorite matrix, has been associated with the entrapment of remnant fluid from a thin layer of interphase. That supports the growth of olivine crystals from the vapor of the solar nebula and constraing on maximun and minimum cooling rates. In this study we present non-destructive techniques that shows carbonaceous material and allows to stimate the chemical and fluid composition of the primitive solar nebula.

A detailed petrographic study of MIA in 18 samples of ordinary and carbonaceous chondrites shows four types of MIA. Were identified in olivine presolar grains grouped into more than two inclusions: (a) MIA-1 consistent with pure glass, (b) MIA-2 consistent with glass and bubble, (c) MIA-3 consistent with glass and variety of minerals, (d) MIA-4 consistent with glass, variety of minerals and bubble. The size of this inclusions range from 1 to 10 microns.

Using a non-destructive thecnique (Raman) shows a spectra obtained in the bubble of melt inclusions showed peaks that were interpreted as the presence of hydrated components and carbonaceous material in chondrites. The spectrum obtained in the glass of melt inclusions in Catalina 008 , shows two peaks that are attributed to bands D and G, correspond to the presence of poly-aromatic carbonaceous material. The study of MIA open a new window to the knowledge of the primitive solar nebula composition. Acknowledge: CONICYT 11170210, FONDEQUIP 170103. 